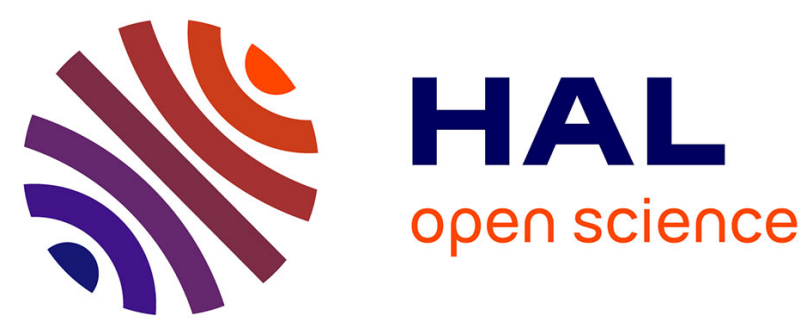

\title{
From Dark Matter to Brittle Fracture
}

P. C. F. Di Stefano, C. Bouard, Sergio Ciliberto, Stéphanie Deschanel, Osvanny Ramos, Stéphane Santucci, A. Tantot, Loïc Vanel, N. Zaim

\section{To cite this version:}

P. C. F. Di Stefano, C. Bouard, Sergio Ciliberto, Stéphanie Deschanel, Osvanny Ramos, et al.. From Dark Matter to Brittle Fracture. Annual Conference on Experimental and Applied Mechanics, Jun 2015, Costa Mesa, United States. pp.183-186, 10.1007/978-3-319-21611-9_23 . hal-01814071

\section{HAL Id: hal-01814071 \\ https://hal.science/hal-01814071}

Submitted on 18 Jan 2022

HAL is a multi-disciplinary open access archive for the deposit and dissemination of scientific research documents, whether they are published or not. The documents may come from teaching and research institutions in France or abroad, or from public or private research centers.
L'archive ouverte pluridisciplinaire HAL, est destinée au dépôt et à la diffusion de documents scientifiques de niveau recherche, publiés ou non, émanant des établissements d'enseignement et de recherche français ou étrangers, des laboratoires publics ou privés.

\section{(ㄷ)(1) $\$$}

Distributed under a Creative Commons Attribution - NonCommerciall 4.0 International 


\title{
From Dark Matter to Brittle Fracture
}

\author{
P.C.F. Di Stefano, C. Bouard, S. Ciliberto, S. Deschanel, O. Ramos, S. Santucci, A. Tantot, L. Vanel, and N. Zaïm
}

\begin{abstract}
Prompted by the intriguing results obtained by some of the rare-event searches looking for the dark matter that may make up the bulk of the matter in the Universe, we have studied brittle fracture as a background in scintillation detectors. Under conditions of ambient temperature and pressure, we have demonstrated a correlation between fracture, acoustic emission, and emission of light in several common scintillators. We present early results from an improved setup. When commissioned, it will provide additional channels to study these phenomena, in controllable atmospheres.
\end{abstract}

Keywords Dark matter • Mechanoluminescence $・$ Scintillator $・$ Brittle Fracture

Our interest in fractoluminescence has its origins in astroparticle physics and particle physics. Since the astronomical observations of F. Zwicky in the 1930s [1], confirmed at many different scales and with many different techniques, astronomers have known that most of the matter in the universe only appears through its gravitational interactions. This is referred to as the mystery of dark matter. Over the years, various attempts have been made to solve this problem. One well-motivated class of candidates is Weakly Interacting Massive Particles (WIMPs) [2]. These particles have the advantage that they appear, independently of astronomy, in many of the extensions to the standard model of particle physics. Evidencing the WIMPs that may be around us would therefore solve a longstanding astrophysical problem, and also open a whole new region of particle physics. The direct detection method is to build a detector and wait for the WIMPs to interact in them. This seemingly simple program is unfortunately a huge experimental challenge because, first, the energies that WIMPs will deposit in a detector are quite small, with a typical scale of the order of $\mathrm{keV}$ to tens of $\mathrm{keV}$. Second, the rate of WIMP interactions is tiny, less than one per month per kilogram of detector, which is tiny compared to typical ambient levels of radioactivity, for instance that of a human body which is of hundreds of radioactive decays per second and per kilogram. Experiments looking for WIMPs therefore take draconian steps to eliminate all forms of background, including careful screening of all materials to ensure radiopurity, specialized shielding against ambient radioactivity, working in deep underground labs to escape cosmic radiation [3], and developing detectors with some form of particle identification to separate the rare signal from the abundant backgrounds. In the late 1990s, the CRESST dark matter search deployed sapphire crystals cooled to $10 \mathrm{mK}$. The detection principle was that particles interacting in them would create a measurable rise in temperature; the attained energy thresholds were less than $1 \mathrm{keV}$ [4]. In the first phase, an unexpectedly high background was encountered. After troubleshooting, this background was identified as originating in small cracks propagating in the crystals because of unintentional indentation at the mounting points, and was eliminated by modifying the contacts between the crystal and its environment. The analysis of these data yielded a very rich data set of precisely calibrated fracture energies down to a values equivalent to a few hundred bonds. Results included demonstrating the Gutenberg-Richter law of fracture energy distribution holds at energies thirty six orders of magnitude below those for a large earthquake, and evidencing Omori's law at similarly small scales [5].

P.C.F. Di Stefano $(\bowtie) \bullet$ C. Bouard $\bullet$ S. Deschanel $\bullet$ A. Tantot $\bullet$ L. Vanel $\bullet$ N. Zaïm

Department of Physics, Queen's University, Kingston, ON, Canada K7L 3N6

e-mail: philippe.distefano@gmail.com

O. Ramos

Institut Lumière Matière, UMR5306 Université Lyon 1-CNRS, Université de Lyon, Villeurbanne Cedex F-69622, France

S. Ciliberto $\bullet$ S. Santucci

Laboratoire de Physique, Université de Lyon, Ecole Normale Supérieure de Lyon,

Centre National de la Recherche Scientifique UMR 5672, 46 Allée d'Italie, Lyon Cedex 07 F-69364, France

S. Deschanel

MATEIS, UMR 5510 CNRS and INSA-Lyon, Université de Lyon, Villeurbanne F-69621, France 
Fig. 23.1 Correlation between fracture, acoustic emission, and emission of light during the rupture of a DCDC sample of [SOUND]) scintillator BGO (from Ref.

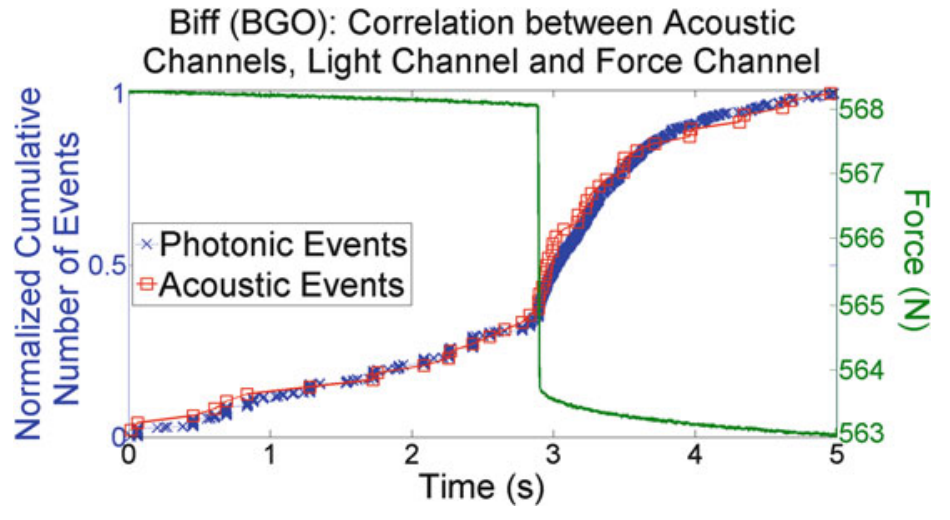

Fig. 23.2 Intact and broken DCDC samples of scintillator BGO exhibiting luminescence under X-ray illumination. Sample dimensions are $20 \times 5 \times 3 \mathrm{~mm}^{3}$. Broken sample shows main fracture along long axis, and a secondary fracture that occurred after the main one when force was kept after main fracture, along one of the short axes

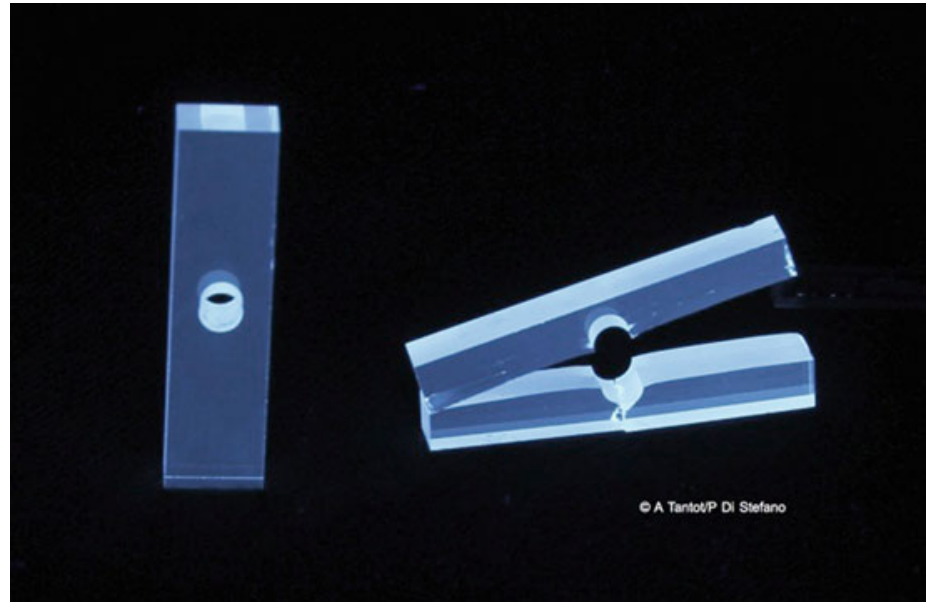

More recently, several dark matter searches have observed intriguing events incompatible with known backgrounds [6-9], in tension with the null results of other experiments [10-16]. We have therefore decided to study fracture as a potential background in rare-event searches, in particular as a source of light in the experiments deploying scintillating detectors. Such devices convert the energy deposited by an interacting particle into light that can be detected by photomultipliers (PMTs) or cryogenic detectors for instance. Detectors of this type are widespread in particle and nuclear physics [17]. For our purpose, after various tests, we converged on scintillator samples compressed in the double-cleavage drilled compression (DCDC) geometry. The samples were $20 \times 5 \times 3 \mathrm{~mm}^{3}$ rectangular prisms, polished to optical quality, with a $1 \mathrm{~mm}$ diameter circular hole drilled perpendicularly in the middle of the $20 \times 5 \mathrm{~mm}^{2}$ face. Under compression along the long axis, cracks formed reproducibly on either side of the hole in the plane parallel to the $20 \times 3 \mathrm{~mm}^{2}$ face. Applied force and acoustic emission (AE) were monitored; the emitted light was also recorded using a PMT. All channels were recorded simultaneously, at $1 \mathrm{Gsample/s}$ for the light channel and at $10 \mathrm{Msample} / \mathrm{s}$ for the others. Our results evidenced a clear correlation between the rupture of various common scintillators (Bi4Ge3O12 aka BGO, ZnWO4 and CdWO4), AE and light emission, in various mechanical configurations at room temperature and ambient pressure as shown in Fig. 23.1 taken from [18]. We have also demonstrated that the spectrum of emitted light is compatible with that of the final stage of the scintillation process, itself linked to transitions in the Bi3+ ion. Because in other cases emission of charged and neutral particles from fracture surfaces has been observed [19], we hypothesise that the mechanism is as follows: as the fracture advances, fractoemitted particles from one surface interact in the other as they do in a scintillator, emitting light. Other mechanisms may be possible, including electric arcs forming, and causing the BGO to luminesce, or some direct stimulation of the Bi3+ ions. Indeed, DCDC BGO samples excited by UV light will luminesce as shown in Fig. 23.2. Using the precise energy calibration of the light channel possible with radioactive sources, we were also able to set a lower limit on the fraction of elastic energy converted to light. We find a value of $3 \times 10^{-5}$, limited by saturation of the light detection channel.

To better understand the mechanisms involved, and avoid saturation of the light detector, we are commissioning an improved setup in the form of a vacuum chamber that can be evacuated or filled with various gases [20]. Other improvements include a distance sensor to monitor the actual compression of the sample, an infrared (IR) camera to track the progression of the fracture in the DCDC sample, filters on the PMT to block IR light and also to reduce saturation from the fracture itself, and a new streaming DAQ that allows resolution of individual photons. An IR picture of a sample being broken in the dark with the PMT running is shown in Fig. 23.3. Offline image processing allows to track the progression of the crack. Lastly, the DAQ controlling the actuator which imposes the force on the sample has been upgraded to allow regulation at a constant 
Fig. 23.3 Propagation of crack in DCDC sample as visualized with camera under IR lighting. IR lighting is chosen so as not to interfere with the PMT which is sensitive to visible wavelengths. Offline data analysis allows to track propagation of crack tip. Sample is squeezed between fixed backstop and movable pushrod. The two cylindrical objects in foreground are $\mathrm{AE}$ sensors

Fig. 23.4 Partial preliminary results from new setup, in vacuum at ambient temperature. Force is ramped up to two different values, allowing the crack to propagate freely the rest of the time. Propagation of the crack is slow, until critical length of fracture is reached, whereupon fracture accelerates. Main failure of sample occurs around $2600 \mathrm{~s}$ from start. A strong correlation between the acoustic emission and the fracture propagation is observed
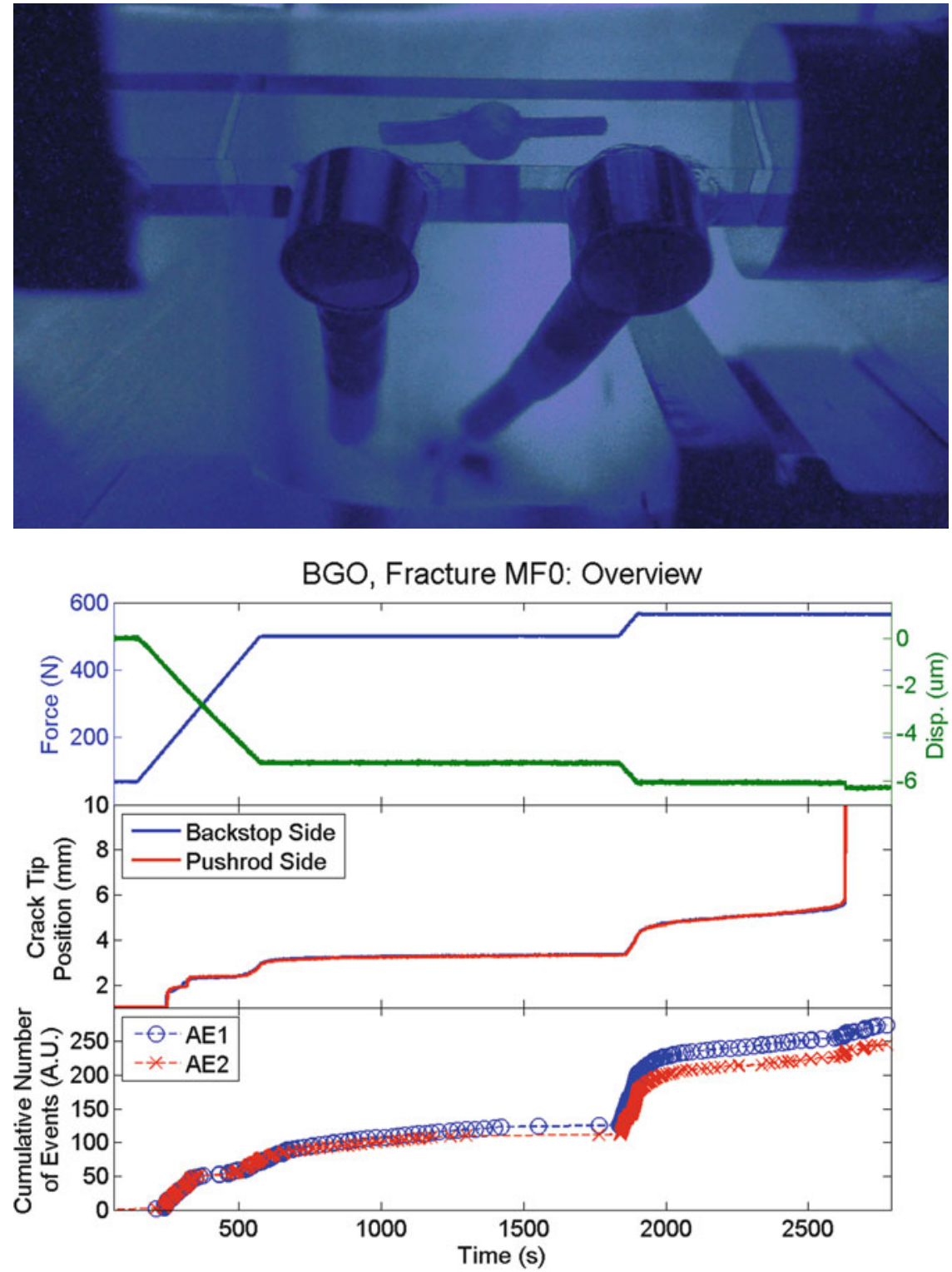

force, enabling studies of sub-critical fracture for instance. Figure 23.4 shows an example from a sample broken at ambient temperature in vacuum. Over the course of nearly an hour, the force was first set at $500 \mathrm{~N}$ while the fracture was allowed to propagate freely and slowly, then ramped up to $565 \mathrm{~N}$, and the fracture propagated freely on its own again, slowly at first before eventually accelerating as expected in the DCDC geometry, and destroying the sample. We observe an excellent correlation between the fracture propagation and the AE. The light channel is not presented for this data set.

In conclusion, we are commissioning an improved setup that will allow us to study brittle fracture in scintillators under various atmospheres with multiple channels: compression distance, crack length, force, acoustic emission and light emission. We expect that this work will be beneficial to development of detectors for rare-event searches, and may lead to insight into fracture mechanisms.

\section{References}

1. Zwicky, F.: Die rotverschiebung von extragalaktischen nebeln. Helv. Phys. Acta 6, 110 (1933)

2. Schnee, R.W.: Physics of the Large and Small. In Csaki, C., Dodelson, S. (eds.) Proceedings of the 2009 Theoretical Advanced Study Institute in Elementary Particle Physics, p. 629. World Scientific, Singapore (2010) 
3. Smith, N.J.T.: The SNOLAB deep underground facility. Eur. Phys. J. Plus 127, 108 (2012)

4. Angloher, G., et al.: Limits on WIMP dark matter using sapphire cryogenic detectors. Astropart. Phys. 18, $43-55$ (2002)

5. Åström, J., et al.: Fracture processes observed with a cryogenic detector. Phys. Lett. A 356, 262-266 (2006)

6. Agnese, R., et al.: Silicon detector dark matter results from the final exposure of CDMS II. Phys. Rev. Lett. 111, 251301 (2013). CDMS Collaboration

7. Aalseth, C.E., et al.: CoGeNT: a search for low-mass dark matter using p-type point contact germanium detectors. Physical Review D 88, 012002 (2013). CoGeNT Collaboration

8. Angloher, G., et al.: Results from $730 \mathrm{~kg}$ days of the CRESST-II dark matter search. Eur. Phys. J. C 22, 1971 (2012). CRESST-II Collaboration

9. Bernabei, R., et al.: First results from DAMA/LIBRA and the combined results with DAMA/NaI. Eur. Phys. J. C 56, 333-355 (2008)

10. Aprile, A., et al.: Dark matter results from 225 live days of XENON100 data. Phys. Rev. Lett. 109, 181301 (2012)

11. Ahmed, Z., et al.: Dark matter search results from the CDMS II experiment. Science 327, 1619-1621 (2010). CDMS II Collaboration

12. Agnese, R., et al.: Search for low-mass weakly interacting massive particles with SuperCDMS. Phys. Rev. Lett. 112, 241302 (2014). SuperCDMS Collaboration

13. Agnese, R., et al.: Search for low-mass weakly interacting massive particles using voltage-assisted calorimetric ionization detection in the SuperCDMS experiment. Phys. Rev. Lett. 112, 041302 (2014). SuperCDMS Collaboration

14. Armengaud, E., et al.: Search for low-mass WIMPs with EDELWEISS-II heat-and-ionization detectors. Phys. Rev. D. 86, 051701(R) (2012). EDELWEISS Collaboration

15. Akerib, D.S., et al.: First results from the LUX dark matter experiment at the sanford underground research facility. Phys. Rev. Lett. 112, 091303 (2014). LUX Collaboration

16. Archambault, S., et al.: Constraints on low-mass WIMP interactions on 19F from PICASSO. Phys. Lett. B 711, 153-161 (2012). PICASSO Collaboration

17. Knoll, G.F.: Radiation detection and measurement, 3rd edn. Wiley, New York (2000)

18. Tantot, A., et al.: Sound and light from fractures in scintillators. Phys. Rev. Lett. 111, 154301 (2013)

19. Langford, S.C., et al.: Simultaneous measurements of the electron and photon emission accompanying fracture of single-crystal MgO. J. App. Phys. 62, 1437 (1987)

20. Tantot, A.: in preparation 